\title{
Vertical motion of ionization induced by the linear interaction of tides with planetary waves
}

\author{
M. Voiculescu ${ }^{1}$ and M. Ignat ${ }^{2}$ \\ ${ }^{1}$ Physics Department, University "Dunarea de Jos", Galati, Romania \\ ${ }^{2}$ Faculty of Physics, University "Al. I Cuza", Iasi, Romania
}

Received: 14 June 2002 - Revised: 4 December 2002 - Accepted: 6 December 2002

\begin{abstract}
Experimental findings have shown that travelling planetary waves modulate the occurrence of midlatitude sporadic-E-layers. Using a simple quantitative model, we analyse the effects of the linear interaction between tides and planetary waves on ion motion. Besides an expected variation of the dumping height, it is found that the boundaries of the oscillations induced by the descending semidiurnal tide are significantly modified by the presence of the planetary wave. The height variations of the ionisation cause planetary wave modulations of the metallic ion content in the background plasma density. This could explain the longterm variation found in the occurrence of strong $E_{s}$ layers. The fact that the dumping height variations are strongly influenced by the tidal phase velocity and amplitude, together with the variability of the metallic ion content, could contribute to the understanding of the sporadic nature of the Elayers.
\end{abstract}

Key words. Ionosphere (Ionosphere-atmosphere interactions; Mid-latitude ionosphere)

\section{Introduction}

Evidence of a strong link between thermospheric dynamics and sporadic-E phenomena have been recently supplemented by the finding that planetary waves (PW) could play a role in the large period wave-like variations found to exist in the occurrence of coherent backscatter echoes (Tsunoda et al., 1998; Voiculescu et al., 1999) and strong sporadic-E occurrence (Voiculescu et al., 1999). In their paper, Voiculescu et al. (2000) tested this postulation and found a fairly good agreement in the long-term periodicities of strong $E_{s}$ and backscatter echo occurrence in conjunction with simultaneously measured neutral wind. A physical explanation of the relationship between the large-scale neutral waves and the formation of intense sporadic-E was first proposed by

Correspondence to: M. Voiculescu

(mirela.voiculescu@ugal.ro)
Shalimov et al. (1999) and developed by Shalimov and Haldoupis (2002). They presented a model of ion accumulation in the centre of a cyclonic vortex, which, according to Holton (1982), accompanies a travelling planetary wave. In a very recent paper, Haldoupis and Pancheva (2002) proved without any doubt that $E_{s}$ parameters are strongly related to planetary waves. They found that the critical frequency of sporadic-E-layers, observed by a large number of ionosonde stations, exhibits a strong 7-days periodicity, occurring concurrently with a large amplitude 7 day westward propagating planetary wave.

The role played by these large-scale neutral waves in the ionospheric plasma was recognized by earlier studies, which related them to the measured long-term periodicities in the $D, E$ and $F$ electron density (Pancheva et al., 1994; Apostolov et al., 1995; Fraser, 1977; Zhou, 1998). In their study, Mathews et al. (1993) noticed a 2-5 day period variation existing in the lower height reached by the ionisation layers at Arecibo and suggested that it might be associated with the action of multiday waves acting on the dumping height. The 2day modulation found in the electron density by Zhou (1998) was attributed to the transportation of metallic ions under the action of the Lorentz force associated to the PW.

Along these lines, we propose in this paper that the PW modulation, found in the occurrence of dense $E_{s}\left(f_{t} E_{s}>\right.$ $5 \mathrm{MHz}$ ) and their top frequency, could be explained by taking into account the modification induced by the action of the alternating eastward and westward direction of the zonal component of this wave on the enhanced ionisation motion. Sporadic E-layers are most likely formed by tides with downward phase velocity (Whitehead, 1961). Due to the increasing collision frequency at low altitudes, the ions cannot follow the descending wind profile and they are dumped at 80$90 \mathrm{~km}$ altitude (Chimonas and Axford, 1968; Mathews and Bekeny, 1979). In the absence of other dynamical or chemical factors, the metallic ions are dumped at altitudes of 85$95 \mathrm{~km}$, where they oscillate with decreasing amplitude. In their numerical computations, Chimonas and Axford (1968) showed that the corkscrew effect of the semidiurnal tide re- 
sults primarily in a layer descent almost at the phase velocity of the wind profile, while Mathews and Bekeny (1979) found that the ion trajectories are consistent with the vertical motions of the layers experimentally observed. MacDougall et al. (2000) found that the lifetime of the metallic ions varies between $10 \mathrm{~h}$ at $100 \mathrm{~km}$ and $10 \mathrm{~min}$ at $90 \mathrm{~km}$, depending also on the electron density. Consequently, a variation of several $\mathrm{km}$ of the dumping height should lead to important variations of the metallic ion density in this height range. For the duration of a semidiurnal period, the PW can roughly be seen as a mean background wind which will either lift or pull down the metallic ions depending on its direction. Therefore, the motion of the ions and the height where they would have been deposited solely by the tide will change. Using a simple theoretical model, we investigate how significant is this variation and what are the implications of this effect on the metallic ion content. To find how the ions are affected by the PW we solve the equation of motion for the ions in the presence of both semidiurnal and planetary wave. The equations and the modelling issues are presented in Sects. 2 and 3, while the results are summarized in Sect. 4. The last two sections are devoted to the discussions and conclusions.

\section{Equations}

The wind shear theory (Whitehead, 1961; Axford, 1963) predicts that a vertical gradient of the horizontal wind provided by a neutral tide leads to convergent vertical ion drifts. The ions gather and form thin layers of enhanced ionisation at the nodes of the wind profile.

The steady-state equation of motion for ions (where the gravitational term is ignored and where only one species of ion is assumed) gives for the velocity vector the well-known formula (e.g. Kelley, 1989):

$$
\begin{aligned}
& \mathbf{V}_{\mathbf{i}}=\frac{1}{1+\beta^{2}}\left\{\mathbf{V}_{\mathbf{n}}+\beta\left(\mathbf{V}_{\mathbf{n}} \times \mathbf{b}\right)+\beta^{2} \mathbf{b} \cdot\left(\mathbf{V}_{\mathbf{n}} \cdot \mathbf{b}\right)\right. \\
& -\frac{k_{B} T_{i}}{m_{i} \Omega_{i}} \beta\left[\frac{\nabla N}{N}+\beta\left(\frac{\nabla N}{N} \times \mathbf{b}\right)+\beta^{2} \mathbf{b} \cdot\left(\frac{\nabla N}{N} \cdot \mathbf{b}\right)\right] \\
& +\frac{e}{m_{i} \Omega_{i}} \beta\left[\mathbf{E}+\beta(\mathbf{E} \times \mathbf{b})+\beta^{2} \mathbf{b} \cdot(\mathbf{E} \cdot \mathbf{b}]\right\},
\end{aligned}
$$

where $\beta=\frac{\Omega_{i}}{v_{i n}}, \mathbf{b}=\mathbf{B} / B$ is the geomagnetic unit vector, $\mathbf{V}_{\mathbf{n}}$ is the neutral wind, $\mathbf{E}$ is the total electric field (polarization plus external field), $\mathbf{N}$ is the plasma density and $\Omega_{i}, v_{i n}$, $m_{i}$ and $T_{i}$ are the ion gyrofrequency, the ion-neutral collision frequency, the ion mass and the ion temperature, respectively. In the following we consider only the combined action of the neutral waves, neglecting the effects of diffusion or electric fields. The diffusion contributes less to the vertical ion velocity and more to the width, peak and lifetime of the layer (Mathews and Bekeny, 1979), while a typical midlatitude zonal electric field of $0.5 \mathrm{mV} / \mathrm{m}$ would modify the ion vertical velocity with less than $1 \mathrm{~m} / \mathrm{s}$.

In a geographical coordinate system, with the $x$-axis pointing east, $y$-axis to north and $z$ positive vertically upwards, a wind $\mathbf{V}_{\mathbf{n}}$ will force the ions to move vertically with the velocity:

$$
\begin{aligned}
& V_{i z}=\frac{1}{1+\beta^{2}} \\
& {\left[\left(1+\beta^{2} \sin ^{2} I\right) V_{n z}+\beta V_{n E} \cos I-\beta^{2} V_{n N} \sin I \cos I\right],}
\end{aligned}
$$

where $V_{n E}$ and $V_{n N}$ are the zonal and the meridional component of the neutral velocity, $V_{n z}$ the vertical component and $I$ the inclination of the geomagnetic field, considered to be $50^{\circ}$ in our calculations.

Sporadic-E layers are formed by a wind shear associated with the semidiurnal tide, while gravity waves with smaller periods contribute to the structuring of the layer (Whitehead, 1989; Mathews, 1998). Since our interest is confined to the influence of planetary waves on the formation of a layer, we will consider the case of a semidiurnal tide, $\mathbf{U}$, of period $T_{s}=12 \mathrm{~h}$, whose components can be approximated with sinusoidal waves:

$$
U_{E, N}(z, t)=U_{0 E, N}(z) \sin \left(\frac{2 \pi z}{\lambda_{z}(z)}+\frac{2 \pi t}{T_{s}}+\varphi_{E, N}\right),
$$

where the wavelength $\lambda_{z}$ and the amplitudes $U_{0 E}, U_{0 N}$ of the zonal and meridional components vary with the altitude (Forbes, 1994) and where $\varphi_{E}$ and $\varphi_{N}$ are the initial phases of the two tidal components. Experimental and theoretical investigations show that the vertical component of the tide is at the most one order of magnitude smaller than the horizontal ones and is not considered in our model.

The other wave acting on the ions is the travelling PW. These waves are interpreted as corresponding to the evanescent solution of the tidal equation of Laplace. They propagate horizontally in a westward direction, but their vertical propagation is forbidden and there is no $z$ variation of amplitude in the lower thermosphere (Forbes, 1994). For the one-dimensional (vertical) model that we use, the waves are seen as time dependent oscillations of the neutral velocity with the period $T_{W}$. The horizontal components of the PW, whose amplitudes and phases are $W_{0 E, N}$ and $\theta_{E, N}$, are described by:

$W_{E, N}(t)=W_{0 E, N} \sin \left(\frac{2 \pi t}{T_{W}}+\theta_{E, N}\right)$.

The effect of the two waves acting simultaneously on the ions in the E-region will sum up to give:

$V_{i z}=V_{i z U}+V_{i z W}$,

where $V_{i z U}(z, t)$ and $V_{i z W}(z, t)$ are respectively the tidal induced and the PW contribution to the vertical ion velocity, obtained by replacing $V_{n}$ with $U$ and $W$, into Eq. (2). After introducing Eqs. (3) and (4) in the Eq. (5), the vertical motion of the ionisation can be determined by solving the equation:

$$
\begin{aligned}
& V_{i z}=\frac{d z}{d t}=\frac{\beta(z) \cos I}{1+\beta^{2}(z)} \\
& {\left[U_{0 E}(z) \sin \left(\frac{2 \pi z}{\lambda_{z}(z)}+\frac{2 p i t}{T_{s}}+\varphi_{E}\right)+W_{0 E} \sin \left(\frac{2 \pi t}{T_{W}}+\theta_{E}\right)\right]+}
\end{aligned}
$$




$$
\begin{aligned}
& +\frac{\beta^{2}(z) \sin I \cos I}{1+\beta^{2}(z)} \\
& {\left[U_{0 N}(z) \sin \left(\frac{2 \pi z}{\lambda_{z}(z)}+\frac{2 p i t}{T_{s}}+\varphi_{N}\right)+W_{0 N} \sin \left(\frac{2 \pi t}{T_{W}}+\theta_{N}\right)\right]}
\end{aligned}
$$

\section{Modelling issues}

Although Eq. (6) can be solved, several simplifying assumptions are utilized, mostly to avoid computational instabilities.

\subsection{The tide}

To conserve the wave energy density, $w=\rho U^{2}$, the tidal amplitude must increase with altitude to compensate the decreasing density (e.g. Kelley, 1989):

$U_{0 E, N}(z)=U_{0 E, N}^{95} \sqrt{\frac{\rho 95}{\rho(z)}}$,

where $U_{0 E, N}^{95}$ and $\rho_{95}$ are the amplitude and the neutral density at $95 \mathrm{~km}$. Theoretically, this is important in the Eregion, but several studies show that this variation is severely reduced by dissipation (Forbes, 1982; Zhou et al., 1997). Moreover, the height where the dumping occurs is relatively low so that the possible variations of the velocity should not affect the final result significantly. Therefore, the amplitude of the wave will be considered constant, $U=50 \mathrm{~m} / \mathrm{s}$. According to the theory of tides, the wavelength is function of altitude (Forbes, 1994):

$\lambda_{z}(z)=\frac{2 \pi H}{\sqrt{\frac{H}{h}\left(\frac{\gamma-1}{\gamma}+\frac{d H}{d z}\right)-\frac{1}{4}}}$,

where $H$ is the scale height, $H=\frac{k_{B} T}{M g}, h$ the equivalent depth and $\gamma$ is the adiabatic constant. The variation of the wavelength is relatively small in this altitude range if the temperature variation with the altitude is considered, and, for the same reasons outlined above, we will consider the wavelength to be constant.

Akchurin et al. (1998) found that the $E_{s}$ parameters are mostly influenced by the $(2,6)$ and $(2,4)$ harmonics of the semidiurnal wave and to a lesser extent, by the $(2,2)$ tide. In the lower thermosphere the first two tides have wavelengths of $30 \mathrm{~km}$ and $50 \mathrm{~km}$, while the $(2,2)$ harmonic has a value of more than $100 \mathrm{~km}$ (Williams, 1996, Forbes, 1994). A medium wavelength of $40 \mathrm{~km}$ will be used to model the effect of both $(2,4)$ and $(2,6)$ modes and the value of $100 \mathrm{~km}$ is attributed to the third wave.

The zonal phase is chosen so that at the initial moment the wind has a node between $110-115 \mathrm{~km}$ altitude, where the $E_{S}$ formation is more efficient. The most propitious structure of the wind for the vertical accumulation of ions has a southeastward component below and a north-westward component above the location of the layer, which means that the zonal and meridional components are opposed in phase. Even if this were an ideal situation, the meridional component is not very effective at low altitudes so that any variation induced by a different meridional phase would be very small. The values of the zonal and meridional phases for the slow descending tides are then:

$\varphi_{E}=-2 \pi / 3 \quad$ and $\quad \varphi_{N}=\pi / 3$,

while for the faster $(2,2)$ tide, they are:

$\varphi_{E}=-\pi \quad$ and $\quad \varphi_{N}=0$.

\subsection{The PW}

Strong and long lasting $E_{S}$ have been found to be present in connection with the quasi two-day wave (QTDW) (Voiculescu et al., 1999, 2000). In addition, the maximum energy in the PW spectrum comes from the short period waves (Jacobi et al., 1998). For this reason the period of the $\mathrm{PW}$ is set to $48 \mathrm{~h}$. The PW is elliptically polarized, with the meridional component larger than the zonal one, especially for low latitudes. As previously stated, when discussing the tide characteristics, any change in the meridional direction leads to negligible effects at altitudes below $110 \mathrm{~km}$. The amplitudes $W_{N}$ and $W_{E}$ are considered equal. Given that experimental measurements have found that usual velocities accompanying a QTDW are between 15 and $25 \mathrm{~m} / \mathrm{s}$ (Clark et al., 1994), we selected the smallest value in order to show that even if the PW amplitude is not large, the mechanism still operates. As for the phase of the PW, the maximum of the zonal component is attained at the noon of odd days (Meek et al., 1996), which means that $\theta_{E, N}=0$.

\subsection{The collision frequency}

Finally, the ion-neutral collision frequency, which is the most important factor in the dumping mechanism, can be expressed using the relation of Banks and Kockarts (1973):

$v_{\text {in }}=$

$\left[\frac{1.80}{\sqrt{A(A+28)}} \cdot n_{N_{2}}+\frac{1.83}{\sqrt{A(A+32)}} \cdot n_{O_{2}} \frac{0.91}{\sqrt{A(A+16)}} \cdot n_{0}\right] \cdot 10^{-14}$.

This relation can be approximated by an analytical expression, considering the fact that neutral densities are exponentially decreasing with increasing altitude:

$v_{i n}(z)=\sum_{j=1}^{3} v_{0 j} \exp \left(\frac{z_{0}-z}{H_{j}(z)}\right)$,

where $H_{j}$ is the scale height of the each neutral species, $H_{j}=\frac{k_{B} T}{M_{j} g}$, and $\nu_{0 j}$ are the ion-neutral collision frequencies of the ions with each neutral species at the reference height $z_{0}$. A realistic approach should consider the altitude dependence of the scale heights due to the important variation of the neutral temperature. As a matter of fact, it is not the collision frequency that is the relevant quantity but its ratio to the ion gyrofrequency, $\beta=\frac{\Omega_{i}}{v_{i n}}$. A simpler analytical description of the altitude dependence of this coefficient is:

$\beta(z)=\beta_{0} \exp \left(-\frac{z_{0}-z}{H}\right)$, 


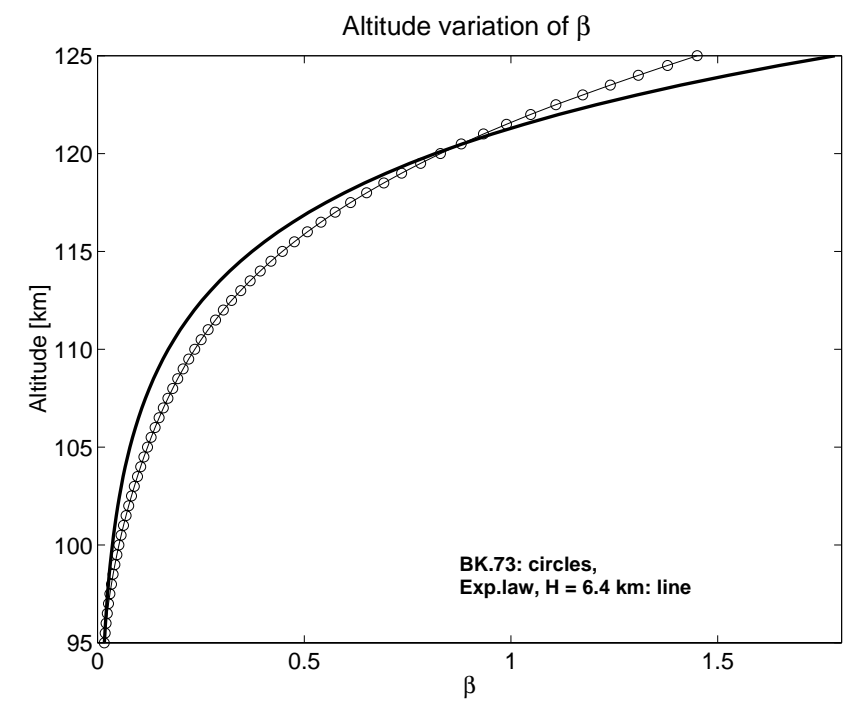

Fig. 1. Altitude dependence of $\beta=\Omega_{i} / \nu_{i}$ for two cases: 1. collision frequency given by Banks and Kockarts, 1973 (circles), with MSIS-90 (15 July) used for the neutral densities; 2 . the same quantity expressed using an exponential law with a constant $H$ (line).

where, for small altitude ranges like those associated with the E-region, the $z$-variable $H$ has been replaced with a fixed one and where $\beta_{0}=\frac{\Omega_{i}}{v_{0}}$, all the contributions of the neutral species being summed up in the value $v_{0}$. Using a simple interpolation program we found that the scale height value giving the best approximation for the altitude dependence of $\beta$ is $H=6.4 \mathrm{~km}$. This can be seen in Fig. 1 where, for Eq. (9), the neutral densities have been taken from the MSIS90 (Hedin, 1991) atmospheric model, run for 15 July.

\section{Results}

The ion trajectories obtained by solving Eq. (6), for an initial position at $120 \mathrm{~km}$, are depicted in Fig. 2, for the duration of a half period of the PW (24 h). The plot in Fig. 2a illustrates the effect of the PW action when the tide descends slower, with $V_{p h} \cong 1 \mathrm{~m} / \mathrm{s}$, while Fig. $2 \mathrm{~b}$ is the result for the faster wave, whose $V_{p h}$ is $2.3 \mathrm{~m} / \mathrm{s}$. Both figures are produced in the same manner, using the same values for $W$ and $U$. The continuous line is the trajectory of the enhanced ionisation carried by the semidiurnal tide alone. The other two curves, marked with triangles, are the trajectories of the same ionisation points under the action of the same tide together with the longer-period wave, during the first half of a PW period (right triangles) and during the second half of a PW period (left triangles). We note, in the beginning, that the dumping heights are slightly higher than those found by Chimonas and Axford (1968). This result is a consequence of the fact that they used a vertical wavelength 3 times larger than the scale height, while this factor is, in our model, 6 and, respectively, 15. This implies a larger phase velocity for "our" tides.

A quick look at the two figures, where equal amplitudes of the tides were used, shows that the faster wave dumps its
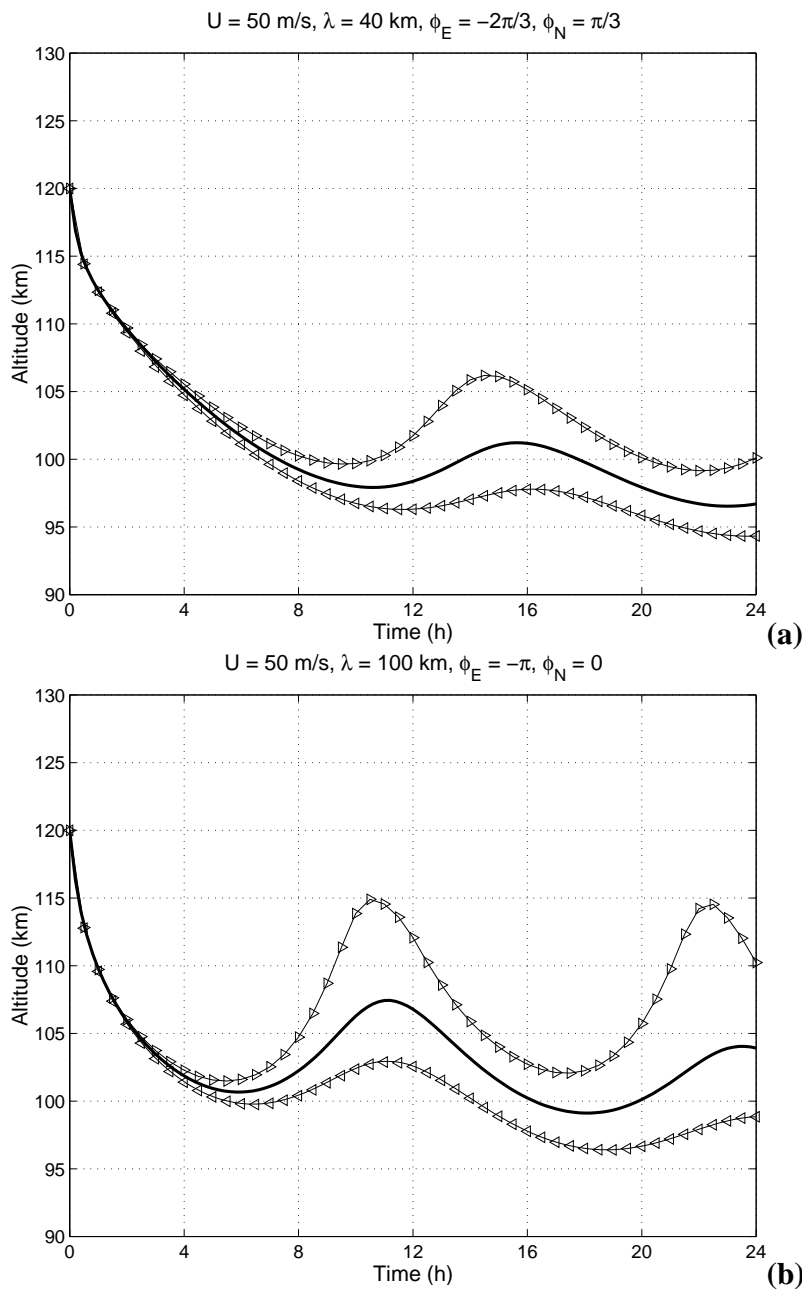

Fig. 2. Ionization trajectories under the action of the tide (thick line), tide + eastward component of the PW (right triangles), tide + westward component (left triangles) for $U=50 \mathrm{~m} / \mathrm{s}$ and $W=$ $15 \mathrm{~m} / \mathrm{s}$. (a) Wavelength $(\lambda)$ of $40 \mathrm{~km}$; (b) wavelength $(\lambda)$ of $100 \mathrm{~km}$.

ionisation at higher heights than the slower one, no matter if the PW component is included or not. This is to be expected, since the ions remain trapped in the nodes of the tide for a longer time when they are carried by a slow moving wave, thus reaching lower heights (Whitehead, 1989; Mathews et al., 1993). It is visible, from all our plots, that the inclusion of the PW is seen mostly in the lower part of the E-region, where the zonal wind controls the motion of the ions. This is in accord with Voiculescu et al. (2000), who found that the long-term periodicities of $E_{s}$ occurrence are associated with those observed in the zonal component of simultaneous wind data.

The height where the ions stop their downward motion is higher when the PW is eastward directed and lower when it overturns. The difference between the two heights seems to be more or less the same, regardless of the wavelength of the tidal wave. In the first plots the two dumping heights are separated by approximately $5 \mathrm{~km}$ after about $12 \mathrm{~h}$ from the beginning of the analysed time interval. The separation 



Fig. 3. The same as for Fig. 2 but for tidal amplitudes, $U$, of $100 \mathrm{~m} / \mathrm{s}$.

Fig. 4. (a) Lifetime of metallic ions function of altitude for three different electron densities. (b) Detail of plot (a), for lifetimes of 0-30h.

\section{Discussion}

is larger, attaining $7 \mathrm{~km}$, for the $(2,2)$ tide. The uppermost heights, where the eastward oriented PW velocity brings the ionisation, depends on the value of the wavelength, thus on the phase velocity. The faster the wave, the higher are the altitudes to which the ions are lifted.

The heights where the ions are carried by the tide depend also on the ratio of the amplitude to the phase velocity, $U / V_{p h}$. When amplitudes of $100 \mathrm{~m} / \mathrm{s}$ were used, the picture changed giving an overall lowering of the heights where the ions stop their vertical descending. This can be seen in Figs. $3 a$ and $b$ where, besides the tidal amplitude, all the other characteristics of the tidal and planetary wave are kept unchanged. An augmentation of the tidal amplitude lowers the dumping height and reduces the PW separation between the uppermost and the lowest altitude. In the case of the $(2,2)$ tide this effect is less dramatic, since the phase velocity of the wave is large when compared with the amplitude.

The modification of the dumping height resulting from the inclusion of other neutral waves was to be expected since, for short time intervals, the long-period wave acts upon the ionisation almost in the same way that a mean wind would do. The eastward component pushes the positive ions up, counteracting the corkscrew effect of the tidal wave, while the opposite one drives the ions to lower heights. It is not only the dumping height variation that is important but also the implications for the metallic ion content.

When the ions reach altitudes close to and below $100 \mathrm{~km}$, the increased neutral density and the low temperature accelerates their transformation into neutral particles. The neutralization of the $\mathrm{Fe}^{+}$ions is governed essentially by the reactions listed in Table 1 (MacDougall et al., 2000 and ref. therein). Figure 4 shows how their lifetime, $\tau=1 / R$, where $R$ is the recombination rate given by MacDougall et al. (2000), varies with the altitude in the low thermosphere for three electron densities, which are considered to charac- 
Table 1. Rate coefficients of reactions leading to the neutralization of the $\mathrm{Fe}^{+}$ions

\begin{tabular}{ll}
\hline Reaction & Rate coefficient $\left(\mathrm{m}^{3} / \mathrm{s}, \mathrm{m}^{6} 7 \mathrm{~s}\right)$ \\
\hline $\mathrm{Fe}^{+}+\mathrm{e}^{-} \rightarrow \mathrm{Fe}+h v$ & $\mathrm{k}_{1}=10^{-18} \mathrm{~m}^{3} / \mathrm{s}$ \\
$\mathrm{Fe}^{+}+\mathrm{O}_{2}+\mathrm{X} \rightarrow \mathrm{FeO}_{2}^{+}+\mathrm{X}$ & $\mathrm{k}_{2}=1.7 \cdot 10^{-41}(300 / T)^{1.86} \mathrm{~m}^{6} / \mathrm{s}$ \\
$\mathrm{Fe}^{+}+\mathrm{N}_{2}+\mathrm{X} \rightarrow \mathrm{FeN}_{2}^{+}+\mathrm{X}$ & $\mathrm{k}_{3}=8.0 \cdot 10^{-42}(300 / T)^{-1.52} \mathrm{~m}^{6} / \mathrm{s}$ \\
$\mathrm{FeO}_{2}^{+}+\mathrm{e}^{-} \rightarrow \mathrm{Fe}+\mathrm{O}_{2}$ & $\mathrm{k}_{4}=3 \cdot 10^{-13}(200 / T)^{0.5} \mathrm{~m}^{3} / \mathrm{s}$ \\
$\mathrm{FeN}_{2}^{+}+\mathrm{e}^{-} \rightarrow \mathrm{Fe}+\mathrm{N}_{2}$ & $\mathrm{k}_{5}=3 \cdot 10^{-13}(200 / T)^{0.5} \mathrm{~m}^{3} / \mathrm{s}$ \\
$\mathrm{FeO}_{2}^{+}+\mathrm{O} \rightarrow \mathrm{FeO}^{+}+\mathrm{O}_{2}$ & $\mathrm{k}_{6}=10^{-16} \mathrm{~m}^{3} / \mathrm{s}$ \\
$\mathrm{FeN}_{2}^{+}+\mathrm{O} \rightarrow \mathrm{FeO}^{+}+\mathrm{N}_{2}$ & $\mathrm{k}_{7}=10^{-16} \mathrm{~m}^{3} / \mathrm{s}$ \\
$\mathrm{Fe}^{+}+\mathrm{O}_{3} \rightarrow \mathrm{FeO}^{+}+\mathrm{O}_{2}$ & $\mathrm{k}_{8}=7.6 \cdot 10^{-16} \exp (-241 / T) \mathrm{m}^{3} / \mathrm{s}$ \\
$\mathrm{FeO}^{+}+\mathrm{O} \rightarrow \mathrm{Fe}+\mathrm{O}_{2}$ & $\mathrm{k}_{9}=7 \cdot 10^{-18} \mathrm{~m}^{3} / \mathrm{s}$ \\
$\mathrm{FeO}^{+}+\mathrm{e}^{-} \rightarrow \mathrm{Fe}+\mathrm{O}$ & $\mathrm{k}_{10}=10^{-13}(200 / T)^{0.5} \mathrm{~m}^{3} / \mathrm{s}$ \\
\hline
\end{tabular}

terize an very dense, a regular and a weak $E_{s}$ layer. The neutral densities and temperature are given by the MSIS-90 model (Hedin, 1991) for $45^{\circ} \mathrm{N}$ and 15 July.

We refer to the days when the zonal PW is eastward as "positive" and to the days when the zoanl PW is westward as "negative". The lowest height where the ions are brought by the $(2,4)$ and $(2,6)$ tides during "positive" days is about $100 \mathrm{~km}$ where they have a lifetime of $1-10 \mathrm{~h}$, depending on the electron densities characterizing the ionisation layer. The modelled trajectory shows that the sheet of enhanced ionisation stops at this height for about $1 \mathrm{~h}$ after which it goes up, reaching an altitude of about $105 \mathrm{~km}$ after $4 \mathrm{~h}$. For typical electron densities (less than $10^{12} \mathrm{~m}^{-3}$ ), the lifetime of ions is large enough; hence, during this time interval, the metallic particles will be maintained in the ionised state. As a result, their uplift will increase the metallic ion density. The maximum density of a stationary layer formed by a sinusoidal neutral wave with amplitude $U$ and wavelength $\lambda$, at a height where the diffusion coefficient is $D$, is given by (Axford, 1968):

$N_{\max }=N_{0} \sqrt{\frac{\beta U_{0} \lambda \sin I}{D}}$.

When the tide reaches the height where the PW has lifted the ions during "positive" days, it will act on a denser plasma generating denser layers. This is valid as long as the electron density is not extremely high so that the lifetime of ions in this height interval is longer than about $4 \mathrm{~h}$. A density of more than $10^{12} \mathrm{~m}^{-3}$, which does not often occur even during times of high $E_{s}$ activity, will probably reduce the PW influence on the metallic ion content. The mean hourly values of the $f_{o} \mathrm{E}_{\mathrm{s}}$ series seen in Fig. 2 of Haldoupis and Pancheva (2002) show that peak frequencies of more than $10 \mathrm{MHz}$ are followed by significantly lower $f_{o} \mathrm{E}_{\mathrm{s}}$, even at times when the 7 day-PW energy seems to be at its maximum. This means that a PW modulation is less likely to be observed in the long-term variation of the occurrence of very strong layers $\left(f o \mathrm{E}_{\mathrm{s}}>10 \mathrm{MHz}\right)$.
The faster semidiurnal wave will deposit the ionisation at higher levels for both eastward and westward directions of the PW although the difference between the two heights remains the same. The ions will be lifted at higher altitudes, where their lifetime increases to several days at $115 \mathrm{~km}$, even for the greatest electron density that we considered. The increase of the metallic content of the plasma will be amplified and will occur over a larger volume than in the previous case. The ion motion is faster; the ions do not have enough time to undergo the neutralization process.

The westward wind associated with the QTDW lowers the dumping height to $95 \mathrm{~km}$ for both tides. There is a lift in this case too, but the height variation is very small $(1-2.5 \mathrm{~km})$. The altitude range where the ions reside is close to $95 \mathrm{~km}$, where the ion lifetime varies over a large range and it is strongly influenced by the electron density. It is very difficult to say how much time the recombination would take, but it is unlikely that the ion metallic density remains unchanged for more than $24 \mathrm{~h}$ (until the velocity associated with the PW changes again to east). Besides, at such low altitudes the collisions are so numerous that the ions cannot be lifted by the horizontal winds involved in our model. Further, at times when the PW is directed westward, the "second" tide will never reach the altitudes where the ions have been brought. Irrespective of whether these ions have been neutralized or not, the wind shear will act on a depleted plasma, providing poorer conditions for a strong layer to form. During these intervals, the ions are swept from the E-region by the combined action of the downward propagating neutral waves and the PW.

The 2-day modulation of the ionisation motion can be seen in Fig. 5, where the ionisation trajectories under the action of successive semidiurnal tides during a 4-day ( 2 cycles) passage of a QTDW are depicted. The two plots summarize the results for the two selected tides having $U=50 \mathrm{~m} / \mathrm{s}$. The dotted rectangles mark the altitude ranges and time intervals where the metallic ion density is enhanced. The density of $E_{s}$ layers generated by the $(2,4)$ and $(2,6)$ tides increases below, approximately, $105 \mathrm{~km}$ in the afternoons of the "positive days". During the "negative" days the $E_{s}$ layer activity should be diminished. If the previous layers have been extremely dense, the increased neutralization rate significant reduces the amount of metallic ions to be lifted; hence the effect is less likely to be observed. Nevertheless, since the plasma density continues to decrease, the PW induced enhancement of the metallic ion content should occur the next "positive" day. The effect seems to be more confined in time when the tide descends faster but the altitude range is larger, extending higher than $115 \mathrm{~km}$. According to our results, the "positive" peak densities of the layers should be larger when formed by the latter tide because the dumping heights are higher and the collection of the ions is more effective than that formed by the former. Hence, the 2-day modulation in the $E_{s}$ parameters should be stronger when the semidiurnal tide has longer wavelengths. The results shown in Fig. 5 emphasize also a 2-day modulation in the lowest height reached by the ions, marked with short dotted lines, which has been 
experimentally observed. Mathews et al. (1993) noticed the existence of $3 \mathrm{~km}$ peak-to-peak wavelike variation with periods of the order of days in the lowest heights where tidal ion layers can be observed. They attributed this effect to the multiday wave modulation of the dumping height or to temperature (and scale height) variation with similar periods.

The modulation of the metallic ion content and of the ionisation motion could explain both the PW modulations of the occurrence of $E_{s}$ layers with $f o \mathrm{E}_{\mathrm{s}}>5 \mathrm{MHz}$ found by Voiculescu et al. $(1999,2000)$ and the 7-day periodicity of the $E_{s}$ critical frequency seen by Haldoupis and Pancheva, (2002). Assuming that all the other factors are constant, since the metallic ion density varies with a certain period, stronger layers will form with the same period. Concomitantly, the ionisation is "maintained" at higher altitudes, favouring an increased occurrence of the $E_{s}$ layers with, again, the same period.

The drop of the dumping heights caused by the increase of the tidal amplitude, seen in Figs. 3a and b, has important effects, especially for the slower 12 -h tides. The separation induced by the opposite direction of the PW is still visible but it is smaller and confined by an altitude range where sporadicE layer formation is not so common. The maximum height reached by the metallic ions is less than $100 \mathrm{~km}$; therefore at higher altitudes the background plasma density is not modified so that the planetary waves cannot cause any modulation of the $E_{s}$ activity. Nevertheless, the effect of the decrease of the phase velocity relative to the tidal amplitude is less important for the faster tide. At $105 \mathrm{~km}$, strong layers could still form and the PW modulation should be observed but the increase of the background density induced by the PW occurs in a vertical region whose extent is smaller. On the other hand, smaller amplitudes, which actually are closer to measured values for the semidiurnal tides, give way to the PW control.

It is known that the E-region is characterized by the joint presence of several tidal modes, with amplitudes and phases varying over a large range (Forbes, 1994; Akchurin et al., 1998). Our results show that both the ionisation motion and the dumping height are modified when tidal parameters are modified. Together with the variability of the metallic ion lifetime at low altitudes, these factors could contribute to the understanding of the sporadic nature of the $E_{s}$ layers.

In the present paper, we did not include other terms, like mean vertical wind or electric field; our intention was to point out the influence of the PW on the dynamics of the ions. When any of those terms is considered, the PW effect on the ion trajectories would presumably be altered. Nevertheless, this modification is strongly dependent on the relative magnitude of the terms and a qualitative analysis cannot give too many indications. A more detailed model, including the altitude variation of the tidal parameters is the subject of a future effort.


Fig. 5. Ionization motion induced by the linear superposition of a tide with $50 \mathrm{~m} / \mathrm{s}$ amplitude and a PW with $15 \mathrm{~m} / \mathrm{s}$ amplitude during 2 cycles of the long-period wave for two tidal wavelengths: (a) $L=$ $40 \mathrm{~km}$; (b) $L=100 \mathrm{~km}$.

\section{Conclusions}

We showed that the variation of the trajectory of enhanced ionisation, induced by the alternating zonal component of a long-period wave, might explain the observed modulation of the strong $E_{s}$ occurrence with periods corresponding to travelling planetary waves. When the PW is eastward, the dumping height is higher and the ions are raised up after a short time. Therefore, the metallic particles are maintained in an ionised state for a longer time, preventing the depletion of the metallic ion density due to downward transportation. The shorter-period waves will lead to the formation of a stronger layer between 100 and $115 \mathrm{~km}$, where the ions brought by the PW have increased the background ionisation. The range of altitude where this effect can be seen depends on the tidal amplitudes and on their phase velocities. The westward directed PW wind forces the ions at lower heights where they recombine relatively fast and where they are kept for a long time. The westward component of the PW enhances the decrease of density induced by the corkscrew effect of the tidal 
wave and the production of dense $E_{s}$ layers is hindered. Consequently, successive $12 \mathrm{~h}$ waves will act on a plasma whose density is modulated with planetary wave periods which, in turn, will lead to a similar modulation of the density and occurrence of the $E_{s}$ layers. This effect is highly dependent on the ratio of the tidal amplitude to the phase velocity.

The PW role in the E-region must be a very complicated phenomenon, requiring more experimental investigations. Theoretical models and experimental work show the presence of non-linear interactions (Teitelbaum and Vial, 1991), which probably have an important contribution to the overall picture of the ionosphere-atmosphere system.

When the model was applied for different phase velocities and amplitudes of the tides, the numerical results showed that the dumping height is greatly influenced by these quantities and on their ratio. The linear interaction between different tides and PW has also different results on the ions motion. This means that in some cases the PW modulation can be stronger than in others depending on what is the dominant mode of the semidiurnal wave. Tides with large phase velocities dump their ionisation at altitudes where the ions survive long enough to maintain the metallic content unchanged, favouring the occurrence of stronger layers. The tides that descend slowly will carry the ions at low altitudes, where, due to the important increase of the recombination rate, they will be rapidly transformed into neutral particles. The metallic percentage of the positive ions will be poorer and $E_{s}$ will be, accordingly, weak.

Our simple model proved also that the ionisation behaviour is significantly changed by any modification of the parameters characterizing the semidiurnal tide. This, together with the large variability of the metallic ion lifetimes could contribute to the understanding the sporadic nature of the occurrence of E-region layers.

Acknowledgement. The authors acknowledge T. Bösinger for valuable comments on the paper. One author (M. V.) thanks the Sodankylä Geophysical Observatory for supporting her visit to the University of Oulu through a Marie Curie research training fellowship granted by the European Community.

Topical Editor M. Lester thanks a referee for his help in evaluating this paper.

\section{References}

Akchurin, A. D., Zykov, E. Yu., Makarov. N. A., Minulin, R. G., Portnyagin, Yu. A., and Sherstyukov, O. N.: The influence of the semidiurnal tide on altitude variations of the sporadic-E layer, Int. J. of Geomagn. and Aeronomy, 1, 89-95, 1998.

Apostolov, E. M., Altadill, D., and Alberta, L.: Characteristics of the quasi-2 day oscillations in the $f o \mathrm{~F} 2$ at northern middle latitudes, J. Geophys. Res., 100, 12 163-12 171, 1995.

Axford, W. I.: The formation and vertical movement of dense ionized layers in the ionosphere due to vertical shears, J. Geophys. Res., 68, 769-779, 1963.

Banks, P. M. and Kockarts, G.: Aeronomy. Part A, Academic Press inc., New York, NY, 1973.
Chimonas, G. and Axford, W. I.: Vertical movement of temperate zone sporadic-E-layers, J. Geophys. Res., 73, 111-117, 1968.

Clark, R. R., Current, A. C., Manson, A. H., Meek, C. E., Avery, S. K., Palo, S. E., and Aso, T.: Hemispheric properties of the two-day wave from mesosphere-lower thermosphere radar observations, J. Atmos. Terr. Phys., 56, 10, 1279-1288, 1994.

Forbes, J. M.: Tidal and planetary waves, in: The upper mesosphere and lower thermosphere, a review of experiment and theory, (Eds) Johnson, R. M. and Killend, T. L., 1994.

Forbes, J. M.: Atmospheric tides 2. The solar and lunar semidiurnal components, J. Geophys. Res., 87, A7, 5241-5252, 1982.

Fraser, G. J.: The 5-day wave and ionospheric absorption, J. Atmos. Sci., 39, 121-124, 1977.

Haldoupis, C. and Pancheva, D.: Planetary waves and midlatitude sporadic-E-layers. Strong experimental evidence for a close relationship, J. Geophys. Res., 107, in press, 2002.

Hedin, A. E.: Extension of the MSIS thermosphere model into the middle and lower atmosphere, J. Geophys. Res., 96, A2, 11591172, 1991.

Holton, J. R.: An Introduction to Dynamic Meteorology, Academic Press, San Diego, California, 1982.

Kelley, M. C.: The Earth Ionosphere: Plasma Physics and Electrodynamics, Academic Press, San Diego, California, 1989.

Jacobi, C., Schminder, R., and Kürschner, D.: Planetary wave activity obtained from long long-period (2-18 days) variations of mesopause regions over central Europe $\left(52^{\circ} \mathrm{N}, 15^{\circ} \mathrm{E}\right)$, J. Atmos. Sol.-Terr. Phys., 60, 81-93, 1998.

MacDougall, J. W., Plane, J. M. C., and Jayachandran, P. T.: Polar cap sporadic-E: part 2, modeling, J. Atm. Solar Terr. Phys., 62, 1169-1176, 2000.

Mathews, J. D. and Bekeny, F. S.: Upper atmosphere tides and the vertical motion of ionospheric sporadic layers at Arecibo, J. Geophys. Res., 84, A6, 2743-2750, 1979.

Mathews, J. D., Morton, Y. T., and Zhou, Q.: Observations of ion layer motions during the AIDA campaign, J. Atmos. Terr. Phys., 55, 3, 447-457, 1993.

Mathews, J. D.: Sporadic E: current views and recent progress, Journal of Atm. and Solar-Terr. Physics, 60, 413-435, 1998.

Meek, C. E., Manson, A. H., Franke, S. J., Singer, W., Hoffmann, P., Clark, R. R., Tsuda, T., Nakamura, T., Tsutsumi, M., Hagan, M., Fritts, D. C., Isler, J., and Portnyagin, Y. I.: Global study of Northern hemisphere quasi 2-day wave events in recent summers near 90 km altitude, J. Atmos. Terr. Phys., 58, 1401-1411, 1996.

Pancheva, D., Alberta, L. F., and de la Morena, B. A.: Simultaneous observations of the quasi-two-day variations in the lower and upper ionosphere over Europe, J. Atmos. Terr. Phys., 56, 43-50, 1994.

Shalimov, S., Haldoupis, C., Voiculescu, M., and Schlegel, K.: Midlatitude E-region plasma accumulation driven by planetary wave horizontal wind shears, J. Geophys. Res., 104, 28 207-28 213 , 1999.

Shalimov, S., and Haldoupis, C.: A model of midlatitude E-region plasma convergence inside a planetary wave cyclonic vortex, Ann. Geophysicae, 20, 1193-1201, 2002.

Teitelbaum, H., and Vial, F.: On tidal variability induced by nonlinear interaction with planetary waves. J. Geophys. Res., 96, 14, 169-14, 1781991.

Tsunoda, R., Yamamoto, M., Igarashi. K., Hocke, K., and Fukao, S.: Quasiperiodic radar echoes from midlatitude sporadic-E and role of the 5-day planetary wave, Geophys. Res. Lett., 25, 951954, 1998.

Voiculescu, M., Haldoupis, C., and Schlegel, K.: Evidence for plan- 
etary wave effects on midlatitude backscatter and sporadic-Elayer occurrence, Geophys. Res. Lett., 26, 1105-1108, 1999.

Voiculescu, M., Haldoupis, C., Pancheva, D., Ignat, M., Schlegel, K., and Shalimov, S.: More evidence for a planetary wave link with midlatitude E-region coherent back scatter and sporadic-Elayers, Ann. Geophysicae, 18, 1182-1196, 2000.

Williams, P. J. S.: Tides, atmospheric gravity waves and traveling disturbances in the ionosphere, in Modern Ionospheric Science, eds. H. Kohl, R. Rüster, K. Schlegel, EGS, 1996.

Whitehead, J. D.: The formation of the sporadic-E-layer in the temperate zones, J. Atm. Terr. Phys., 20, 49-58, 1961.

Whitehead, J. D.: Recent work on mid-latitude and equatorial sporadic-E, J. Atm. Terr. Phys., 51, 401-424, 1989.

Zhou, Q. H.: Two-day oscillation of electron concentration in the lower ionosphere, J. Atm. Solar Terr. Phys., 60, 1669-1677, 1998.

Zhou, Q. H., Sulzer, M. P., and Tepley, C. A.: An analysis of tidal and planetary waves in the neutral winds and temperature observed at low latitude E-region heights, J. Geophys., Res., 102, A6, 11 491-11505, 1997. 